\title{
Factores explicativos de la distribución espacial de la temperatura del aire de verano en Santiago de Chile $^{1}$
}

\author{
Pamela Smith ${ }^{2}$ y Hugo Romero 3
}

\begin{abstract}
RESUMEN
Santiago de Chile presenta importantes diferencias en la distribución de las temperaturas del aire en el interior de la ciudad debido a que un conjunto de factores explicativos, como el incremento de las tasas de impermeabilización y la reducción de la vegetación han generado islas de calor que se acentúan y generalizan en las noches de verano y que conforman un complejo y heterogéneo mosaico de paisajes urbanos que afectan la calidad ambiental y de vida de sus habitantes. Sobre la base de redes de información climático-urbana que asocian datos provenientes de estaciones fijas, transectos móviles e imágenes satelitales termales, se han estimado relaciones espaciales significativas que han permitido la construcción de mapas que contribuyen a explicar la variabilidad temporal y espacial de las temperaturas atmosféricas y con ello sugerir la adopción de medidas de mitigación y adaptación tanto ante los fenómenos de macro como de mesoescala.
\end{abstract}

Palabras clave: Clima urbano, distribución espacial de temperaturas, modelos de regresión

\begin{abstract}
The city of Santiago (Chile) shows significant differences in the distribution of air temperatures within the city due to a set of explanatory factors, such as increased proportions of impermeable surfaces and a reduction in vegetation. This has in turn generated heat islands, which are accentuated and generalized in the summer nights, creating a complex and heterogeneous mosaic of urban landscapes that affect the environment and quality of life of its inhabitants. Based on networks of urban-climate information that link data from fixed stations, mobile transects and thermal satellite imagery, we estimate significant spatial relationships that have allowed us to construct maps that help to explain the temporal and spatial variability of atmospheric temperatures, and thus to suggest measures for mitigating and adapting to the phenomena at both the macro- and meso-scale.
\end{abstract}

Key words: urban climate, spatial distribution of temperatures, regression models

1 Proyectos FONDECYT No 1100657 y No 1130305 Artículo recibido el 23 de enero de 2015, aceptado el 11 de septiembre de 2015 y corregido el 21 de diciembre de 2015.

\footnotetext{
2 Departamento de Geografía, Universidad de Chile (Chile). E-mail: pesmith@uc.cl

3 Departamento de Geografía, Universidad de Chile (Chile). E-mail: hromero@uchilefau.cl
} 
Existen diferentes definiciones de clima. La más tradicional lo considera como la descripción y clasificación de los lugares de acuerdo al estado medio de la atmósfera, teniendo en cuenta complementariamente, la variabilidad temporal en el comportamiento de diversos indicadores del calor, contenido de humedad atmosférica, presencia y velocidad de la ventilación (Linés, 1996). Otras definiciones enfatizan su multiescalaridad espacial y temporal (relacionando los procesos de escala global con los fenómenos observados a escala regional y local y los eventos registrados en el corto, mediano y largo plazo) y más recientemente en su construcción cultural, aproximándose crecientemente a lo que se podría definir como clima social o socioclima (Hulme, 2015).

El clima de las ciudades presenta ciertas particularidades que lo tornan diferente del registrado en zonas rurales y que se relacionan principalmente con las características que presenta la superficie terrestre en las áreas urbanizadas (Oke, 1987; Arnfield, 2003). El estudio de los climas urbanos ha despertado un interés creciente debido a que la mayor parte de la población nacional y mundial reside en ciudades y por ello participa de las condiciones atmosféricas especiales que se generan en estas áreas (presencia de islas de calor y frío, menor humedad atmosférica debido a la sustitución de las superficies naturales y verdes por cemento) o sufre directamente la falta de confort térmico y los efectos de la contaminación atmosférica.

La complejidad de los procesos que generan un clima urbano crece a medida que se incrementa la escala de análisis, y van adquiriendo una importancia creciente aspectos como los usos y coberturas del suelo, la morfología de la ciudad y la materialidad de sus construcciones (Stewart \& Oke, 2009). Las coberturas impermeabilizadas difieren en sus formas de absorber, almacenar y emitir energía (Grimmond \& Oke, 1998; Kusaka, 2008). Los procesos de mezcla turbulenta son mayores en las áreas edificadas (Sendiña \& Pérez, 2006), y respecto al comportamiento de la temperatura atmosférica, se ha demostrado que en la tarde y tras la puesta de sol, las ciudades registran temperaturas más elevadas, que alcanzan su máximo en las islas de calor que generalmente corresponden a superficies de mayor densidad de ocupación humana. Los factores que explican este proceso se relacionan con la eliminación de la vegetación y de los suelos permeables, que generan una mayor evaporación y humedad relativa del aire (Coutts et al., 2007), la menor admitancia térmica de los elementos de las superficies construidas (Grimmond \& Oke, 1998), la mayor rugosidad introducida por los edificios (Oke, 1987; Johansson, 2006), todas propiedades que interactúan con las características orográficas de los sitios en que se instalan o desarrollan las ciudades, que en general, intervienen en la movilidad y mezcla de las masas de aire, como sucede con las cuencas cerradas (Jo et al., 2001; Cuadrat et al., 2005), y con las condiciones atmosféricas sinópticas, que pueden favorecer la ocurrencia de inversiones térmicas o de tiempos meteorológicos caracterizados principalmente por vientos débiles (Georgakis et al., 2010).

Entre los factores mencionados, cabe destacar que la presencia de superficies vegetadas es decisiva para la mitigación de las islas de calor urbano, control de la contaminación y producción de sombra, todos los cuales constituyen servicios ambientales cuya mantención y fortalecimiento debería ser uno de los objetivos más relevantes de la planificación urbana ambientalmente sustentable (Oke, 1982; Honjo et al. 2003). Por ejemplo, la sombra generada por los árboles enfría el aire y reduce entre un 10 y $30 \%$ la velocidad del viento (Bolund \& Hunhammar, 1999), a lo que se debe agregar la capacidad de las áreas verdes, para transformar el calor sensible en calor latente (Flocks et al., 2011). Por otra parte, la impermeabilización de los suelos, al inhibir la infiltración del agua, reduce la evapotranspiración (Pauleit et al., 2005), la que llega a ser entre un 30 y $40 \%$ menor que en áreas permeables (Arnold \& Gibbons, 1996). Parques y jardines se comportan como "islas de frescor" y generan un fenómeno Ilamado "brisas de parque" que se desarrollan especialmente durante las noches, contribuyendo a enfriar los espacios construidos contiguos y a limpiar la atmósfera con el desplazamiento del aire desde las áreas frías a las más cálidas (Nowak \& McPherson, 1993). Lo mismo ocurre con la presencia de cuerpos de agua (Molina, 2007; Sarricolea, 2009). 
La altura del sitio de la ciudad, así como la posición topológica (que se ubique en el fondo o sobre las laderas de una cuenca), determina la cantidad de insolación recibida a distintas horas del día (Cuadrat et al. 2005; Jo et al., 2001; Sarricolea \& Romero, 2009). La rugosidad urbana, un índice referido a la altura de las edificaciones, a escala del dosel, atrapa la radiación de onda larga durante la noche, reduciendo las velocidades de enfriamiento que tendrían los objetos urbanos en ausencia de obstáculos. Las estructuras urbanas modifican además, el acceso de luz solar durante el día y la dirección y velocidad del flujo del viento a nivel de las calles (SprokenSmith \& Oke, 1999; Eliasson, 1999; Rohinton, 1999 y Honjo et al., 2003).

La ciudad de Santiago de Chile concentra alrededor del $40 \%$ de la población total del país, y entre los años 1980 y 2010 ha duplicado su superficie urbanizada. Respecto a sus climas urbanos se han desarrollado estudios sistemáticos que relacionan los cambios de usos y coberturas de suelos asociados a la rápida urbanización con las transformaciones de las temperaturas y generación de islas de calor, la covariación entre estas y sus espacios sociales y las concentraciones de contaminantes atmosféricos (Romero y Opazo, 2011). Otros estudios han abordado la variabilidad espacial de la temperatura de emisión superficial a meso escala, para distintos usos de suelo y tipos de construcciones durante el día (Peña y Romero, 2006; Irarrázabal, 2011) y la noche (Sarricolea, 2012), mediante análisis de imágenes satelitales y levantamientos obtenidos por transectos móviles (Molina, 2007), estudiando los factores que explican su comportamiento y distribución (Sarricolea, 2008). Sin embargo, no existen antecedentes que informen sobre la variabilidad espacio-temporal de las temperaturas atmosféricas urbanas, y que proporcionen información susceptible de ser considerada en la planificación urbana. Considerando lo anterior, esta investigación pretende contribuir con la generación de un modelo espacial para estimar la distribución de las temperaturas atmosféricas al interior de la ciudad de Santiago, con la finalidad de:

- Sistematizar, sintetizar y relacionar los datos de temperatura atmosférica obtenidos desde estaciones fijas y móviles distribuidas en diversas áreas urbanas.

- Identificar los principales factores ambientales y urbanos que contribuyen a explicar la variabilidad espacio-temporal de la temperatura atmosférica, cuya consideración sería fundamental para ser incorporados en la planificación urbana sustentable de la ciudad.

- Superar las limitaciones de representatividad de los datos meteorológicos recopilados al interior de la ciudad debido a la complejidad y elevada variabilidad espacio-temporal del mosaico de paisajes urbanos, mediante la aplicación de modelos estadísticos que faciliten su comprensión e interpretación.

\section{Metodología}

Este estudio ha comprendido el análisis de las temperaturas atmosféricas de la ciudad de Santiago, abarcando la escala vertical del dosel urbano (Urban Canopy Layer según Oke, 2010) y la horizontal de mesoescala, cubriendo la mancha urbana de la ciudad (Urban Boundary Layer según Oke, 2010), sobre la base de datos recopilados en la estación de verano del año 2009. Se evaluó la normalidad de los registros comparando el promedio y desviación estándar de los datos obtenidos de estaciones meteorológicas fijas con el promedio de temperatura del aire de los últimos 30 años (Villarroel et al., 2006).

Las temperaturas urbanas se espacializaron utilizando sistemas de información geográfica, específicamente módulos de las versiones ArcGis 10 e Idrisi TAIGA 16. La generación de modelos que explican el comportamiento horario y distribución espacial de las temperaturas se realizó mediante análisis de regresión múltiple. Finalmente, con la finalidad de validar las estimaciones obtenidas para horas de la mañana, tarde y noche, se compararon con los datos recogidos en terreno mediante la realización de transectos móviles.

\section{Obtención de datos de temperatura atmosférica}

Los datos analizados en este trabajo corresponden a treinta estaciones meteorológicas fijas (Figura $\mathrm{N}^{\circ} 1$ ), facilitados por el Centro Nacional del Medio Ambiente (CENMA) de la Universidad de Chile, el Departamento de Geofísica de la Universidad de Chile, la Secretaría Ministerial de Salud de la Región Metropolitana de Santiago a través de la Red 
de Monitoreo de la Contaminación Atmosférica del Área Metropolitana (MACAM), el Departamento de Física de la Universidad de Santiago de Chile, la Dirección General de Aguas (DGA) y el Instituto Nacional de Investigaciones Agropecuarias (INIA), que se deben sumar a los provenientes de instrumentos de medición HOBO instalados en Santiago por el Laboratorio de Medio Ambiente y Territorio del Departamento de Geografía de la Universidad de Chile. Para la validación de las estimaciones estadísticas se recurrió a datos de temperatura atmosférica obtenidos tanto desde puntos fijos de observación como desde transectos móviles de mediciones realizadas los días 20 y 21 de enero del 2011, durante la mañana (10:30 horas), tarde (14:30 horas) y noche (20:30 horas), cuyos recorri- dos abarcaron los diferentes usos y coberturas de suelo, atravesando la ciudad de norte a sur y de oriente a poniente, incluyendo las áreas rurales periféricas.

Se realizó un control de calidad, mediante consultas al personal responsable de la manipulación de los instrumentos y de los metadatos así como de la continuidad de los registros y calibración de instrumentos. Para la homogenización de series, se contrastó gráficamente el comportamiento anticíclico de la temperatura atmosférica con la humedad relativa, utilizándose además gráficos de caja para observar la variación de los datos. Aquellos muy dispersos fueron identificados utilizando el módulo de detección de anomalías del software IBM SPSS Statistics 19.

Figura $N^{0} 1$

Localización de las estaciones fijas y de las consideradas por los transectos móviles de medición de las temperaturas urbanas en la ciudad de Santiago.

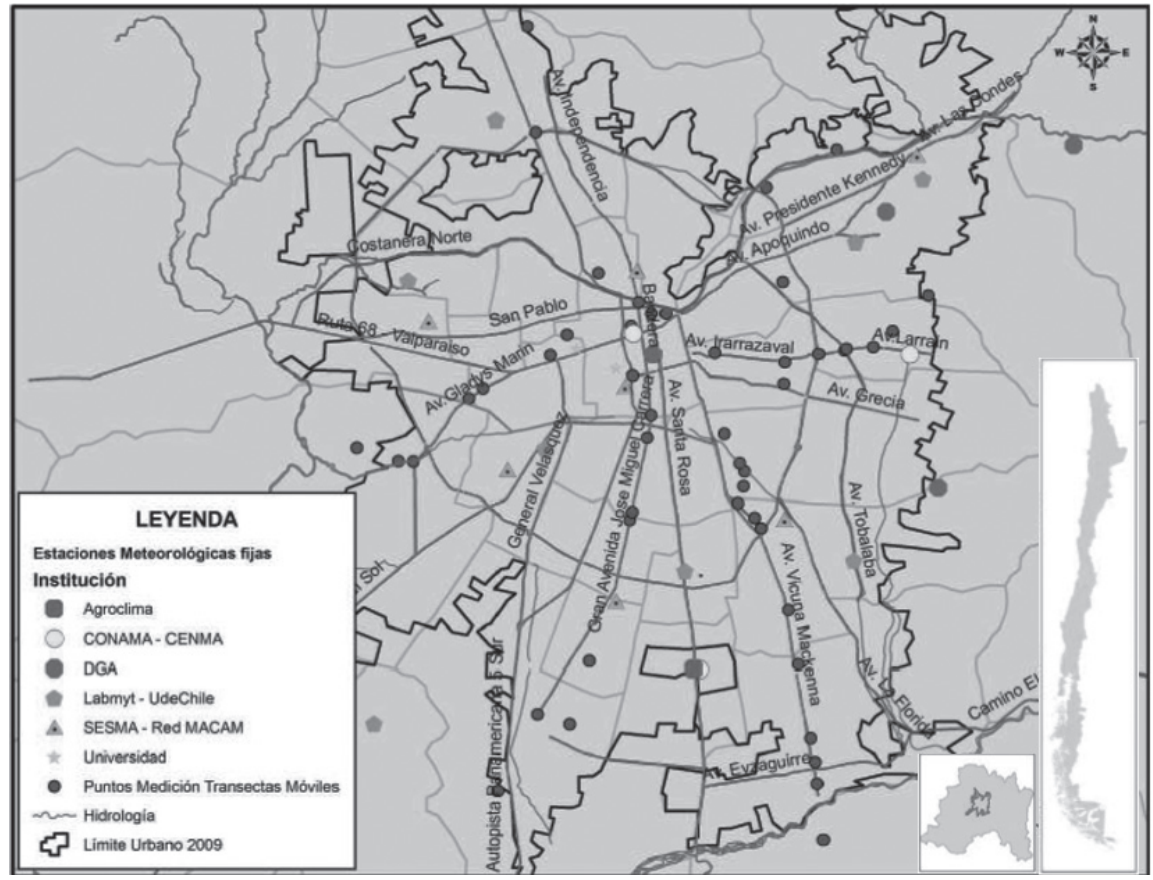

Fuente: Elaboración propia. 


\section{Selección de variables explicativas y representación espacial de las mismas}

Para la selección de las variables relevantes, se consideraron las investigaciones realizadas por los autores mencionados en el Cuadro $N^{\circ} 1$. Para la representación de los factores geográfico-urbanos que pemiten explicar la distribución espacial de las temperaturas, se utilizaron coberturas elaboradas por el Proyecto de Ordenamiento Territorial Ambientalmente Sustentable de Santiago (OTAS, 2002), y datos e informaciones resultantes del tratamiento digital de imágenes satelitales
LANDSAT y ASTER en los Sistemas de Información Geográfica Idrisi Andes y Arcgis 9.2.

Se analizaron once imágenes captadas por el satélite LANDSAT 5 para el año 2009, de acceso libre en la página web del Instituto Nacional de Pesquisas Espaciales (INPE) de Brasil. Dos de ellas corresponden al período estival (12 de febrero y 16 de marzo). Se escogió la primera de estas fechas debido a su mejor visibilidad. Adicionalmente se contó con una imagen ASTER del 4 de febrero, proporcionada por el Helmholtz Centre for Environmental Research - UFZ, Leipzig, Alemania.

\section{Cuadro $\mathrm{N}^{\circ} 1$}

Variables explicativas de la distribución de las temperaturas urbanas según diversos autores

\begin{tabular}{|c|c|}
\hline Variable & Autor \\
\hline Cobertura Vegetal & $\begin{array}{c}\text { Oke (1982); Nowak \& MacPherson (1993); Honjo et al. } \\
\text { (2003); Coutts (2007); Nyuk \& Kardinal (2008) }\end{array}$ \\
\hline Superficies Impermeables & $\begin{array}{c}\text { Arnold \& Gibbons (1996); Pauleit, et al. (2005); Stewart \& } \\
\text { Oke (2009) }\end{array}$ \\
\hline Elevación topográfica & Jo et al. (2001); Cuadrat et al. (2003) y (2005) \\
\hline Orientación de laderas & Sarricolea \& Romero (2009) \\
\hline Rugosidad & Oke (1987); Eliasson (1999); Rohinton (1999); Honjo et al. \\
(2003); Johansson (2006)
\end{tabular}

Fuente: Elaboración propia.

Tanto la cobertura vegetal como las tasas de impermeabilización, correspondientes a porcentajes de superficie de los suelos, se obtuvieron a partir de una clasificación supervisada de la superficie terrestre, a escala de sub píxel de las imágenes satelitales. La altura se obtuvo a partir de un modelo digital de elevación, a partir del cual se calculó también la exposición de laderas. La rugosidad urbana, que corresponde a la altura de las edificaciones del año 2008, fue proporcionada por el Servicio de Impuestos Internos y consiste en un valor promedio por manzana censal.

La temperatura de emisión superficial de la imagen Landsat fue calculada en el pro- grama IDRISI a partir del procesamiento de su banda termal, sobre la base de los valores de radiancia, incorporando una emisividad inicial de 0.98. La imagen ASTER contaba ya con los resultados del cálculo de temperatura de emisión superficial desde su fuente de origen, habiendo sido obtenida de acuerdo a la metodología planteada por Wong et al. (2009).

Finalmente, para conocer la distribución espacial diaria de las temperaturas atmosféricas, se realizó una interpolación de sus valores horarios, a través del método Kriging, considerando los datos aportados por las 
distintas estaciones fijas distribuidas en la ciudad.

\section{Elaboración de modelos de regresión y análisis de consistencia estadística}

Los días seleccionados para construir el modelo de regresión (04 y 12 de febrero de 2009) cuentan con imágenes satelitales que permiten relacionar la temperatura atmosférica con los factores ambientales y urbanos seleccionados, mientras que los días 20 y 21 de enero se realizaron las campañas de medición de temperatura, y se contó por tanto, con 49 puntos asociados a los transectos móviles, que si bien no fueron incluidos en el modelo y su representación espacial, sí lo hicieron en su validación (Molinero, 2002), permitiendo la comparación entre los valores estimados y observados.

Para la elaboración de los modelos, se consideraron las correlaciones entre la temperatura atmosférica, como variable dependiente y un conjunto de factores explicativos (Cuadro $N^{\circ} 1$ ). En aquellos casos en que la distribución de los datos fue normal, se utilizó el Coeficiente de Correlación de Pearson, y en los restantes, se aplicó el Coeficiente de Correlación de Spearman. Solo fueron retenidas en los modelos de regresión múltiple, aquellas variables que alcanzaron una correlación estadística significativa con la temperatura atmosférica. Para verificar la consistencia estadística de los modelos, se consideró el valor de las pruebas $\mathrm{F}$ y t, y se realizó una validación cruzada del valor del Coeficiente de Determinación $\mathrm{R}^{2}$.

\section{Diagnóstico de los modelos de regresión}

El diagnóstico del modelo de regresión contrasta los datos recogidos en terreno por medio de transectos móviles (datos observados o empíricos) con aquellos que se obtienen de su aplicación (datos estimados o modelados).

\section{Caracterización climática del área de estudio}

El análisis de la normalidad de los registros realizados durante el año 2009, señala que tanto el promedio de los datos anuales como del periodo estival, presentaron anomalías positivas de $0,4^{\circ} \mathrm{C}$ respecto al promedio de la serie 1989 - 2009. En ambos casos el promedio no superó una desviación estándar, pudiendo considerarse como un año y verano normales. A pesar que las estaciones utilizadas se localizan sobre coberturas y usos de suelo diferentes, incluyendo algunas fuera del área urbana, el comportamiento de sus registros difirió en los valores extremos, máximos y mínimos diarios, pero no en las tendencias observadas durante el año. El mes de marzo del período de verano 2009 fue anormalmente cálido y seco, a diferencia de los meses de enero y febrero, que presentaron un comportamiento normal de sus temperaturas.

El comportamiento del viento tanto en intensidad como dirección predominante, mostró diferencias entre las tres estaciones consideradas (Independencia, Tobalaba y Pudahuel), siendo esta última la que registró las mayores velocidades. La máxima intensidad de la velocidad del viento se registró siempre durante horas de la tarde, mientras que la mínima lo hizo durante la noche.

El día 21 de enero se observó la presencia y paso de una dorsal en altura en fase con una vaguada costera en superficie, condición típicamente asociada al calentamiento de los niveles bajos de la tropósfera. El tiempo atmosférico de ese día mostró una variación de cielo despejado a parcial nublado explicado por la disminución de la estabilidad atmosférica originada por el paso de la dorsal y la posterior aproximación de una vaguada en altura.

\section{Modelo de Regresión de temperatura}

Las variables escogidas corresponden a altura, temperatura de emisión superficial, superficie impermeable, cobertura vegetal, y la interpolación de las temperaturas atmosféricas. De las variables escogidas, la altura queda excluida en el modelo de las 22:00 horas ya que no se encuentra relacionada significativamente con el comportamiento de la temperatura atmosférica. La temperatura de emisión superficial también fue descartada a las 22 horas debido a que corresponde a horas de la mañana.

La altura del sitio y la cobertura vegetal de los suelos se relacionan inversamente con la distribución espacial de la temperatura del aire (Cuadro $\mathrm{N}^{\circ} 2$ ), ratificando las informaciones aportadas por Jo et al., 2001; Nyuk \& Kardinal, 2008). 
Cuadro $\mathrm{N}^{\circ} 2$

Modelos de regresión de la distribución espacial de la temperatura del aire en Santiago

\begin{tabular}{|c|c|c|}
\hline Hora & Modelo de regresión & $\mathrm{R}^{2}$ \\
\hline Mañana & $\begin{array}{r}\text { Temperatura del aire }\left({ }^{\circ} \mathrm{C}\right)=-(\text { Altura } * 0,001)-(\text { Vegetación } * \\
0,005)+\left(\begin{array}{l}\text { Impermeabilización } * 0,006)+(\text { Interpolación } * 1,424)- \\
\text { (Temperatura Superficial } * 0,077)-9,923\end{array}\right.\end{array}$ & 0,873 \\
\hline Tarde & $\begin{array}{r}\text { Temperatura del aire }\left({ }^{\circ} \mathrm{C}\right)=-(\text { Altura } * 0,001)-(\text { Vegetación } * \\
0,015)+\left(\begin{array}{l}\text { Impermeabilización } * 0,004)+(\text { Interpolación } * 1,272)- \\
\text { (Temperatura. Superficial * } 0,006)-8,269\end{array}\right.\end{array}$ & 0,969 \\
\hline Noche & $\begin{array}{r}\text { Temperatura del aire }\left({ }^{\circ} \mathrm{C}\right)=-(\text { Vegetación } * 0,0058)+(\text { Impermea- } \\
\text { bilización } * 0,0064)+(\text { Interpolación } * 1,019)-0,435\end{array}$ & 0,997 \\
\hline
\end{tabular}

Fuente: Elaboración propia.

El Cuadro $\mathrm{N}^{\circ} 3$ indica que en los tres momentos del día la presencia de la vegetación disminuye la temperatura de las áreas urbanas, en valores que oscilan entre 0,5 y $1,5^{\circ} \mathrm{C}$.
La altura reduce levemente la temperatura. Por el contrario, las superficies impermeables contribuyen a aumentarla.

Cuadro $\mathrm{N}^{\circ} 3$

Efecto de la vegetación, impermeabilización y altura sobre la temperatura del aire en Santiago

\begin{tabular}{|c|r|r|r|}
\hline & $100 \%$ cobertura vegetal & $\begin{array}{c}100 \% \text { superficie im- } \\
\text { permeable }\end{array}$ & $\begin{array}{c}\text { Diferencia de temperatura } \\
\text { cada 100 metros de altura }\end{array}$ \\
\hline Mañana & $-0,5^{\circ} \mathrm{C}$ & $+0,6^{\circ} \mathrm{C}$ & $-0,1^{\circ} \mathrm{C}$ \\
\hline Tarde & $-1,5^{\circ} \mathrm{C}$ & $+0,4^{\circ} \mathrm{C}$ & $-0,1^{\circ} \mathrm{C}$ \\
\hline Noche & $-0,58^{\circ} \mathrm{C}$ & $+0,64{ }^{\circ} \mathrm{C}$ & No aplica \\
\hline
\end{tabular}

Fuente: Elaboración propia.

El Cuadro $N^{\circ} 4$ muestra la temperatura del aire que resulta de considerar diferentes arreglos espaciales entre cobertura vegetal y superficies impermeables. En el ejemplo expuesto se parte de una temperatura inicial igual a $30^{\circ} \mathrm{C}$. La diferencia más significativa se registra durante la tarde, cuando, por ejemplo, al pasar de un $100 \%$ a un $75 \%$ de superficies impermeables se obtiene una reducción de $0,5^{\circ} \mathrm{C}$. Una sustitución de las cubiertas de suelos desnudos por superficies vegetadas debería reducir más significativamente las temperaturas. 
Cuadro $\mathrm{N}^{\circ} 4$

Efectos de la cobertura vegetal y superficies impermeables sobre una temperatura atmosférica igual a $30^{\circ} \mathrm{C}$ en la ciudad de Santiago

\begin{tabular}{|r|r|r|r|r|}
\hline \multicolumn{1}{|c|}{$\begin{array}{l}\text { Cobertura } \\
\text { Vegetal }\end{array}$} & $\begin{array}{c}\text { Superficie } \\
\text { Impermeable }\end{array}$ & \multicolumn{1}{c|}{ Mañana } & \multicolumn{1}{c|}{ Tarde } \\
\hline \multicolumn{2}{|c|}{ Porcentaje $(\%)$} & \multicolumn{3}{|c|}{ Temperatura $\left({ }^{\circ} \mathrm{C}\right)$} \\
\hline 100 & 0 & 29,5 & 28,5 & 29,4 \\
\hline 75 & 25 & 29,78 & 28,99 & 29,73 \\
\hline 50 & 50 & 30,05 & 29,45 & 30,03 \\
\hline 25 & 75 & 30,3 & 29,93 & 30,34 \\
\hline 0 & 100 & 30,6 & 30,4 & 30,64 \\
\hline
\end{tabular}

Fuente: Elaboración propia.

\section{Validación estadística del modelo}

En los tres modelos de regresión construidos, el valor del coeficiente de determinación $\mathrm{R}^{2}$ (Cuadro $\mathrm{N}^{\circ}$ 2) se encuentra cercano a 1 , lo que les otorga validez estadística, además de presentar una validación cruzada inferior a 0,1 , garantizando ambos su nivel de confiabilidad en los tres horarios diarios (Molinero, 2002).

\section{Representación espacial del modelo: mapa térmico de Santiago en el verano de 2009}

Además de las diferencias que existen entre la ciudad y su entorno rural, la Figura $\mathrm{N}^{\circ}$ 2 demuestra que el área urbana no es térmicamente homogénea a esta hora del día. Las temperaturas más altas a las 9 de la mañana alcanzan hasta los $23,2^{\circ} \mathrm{C}$ y se concentran al sur de la ciudad, entre las avenidas Vicuña Mackenna y Gran Avenida, lo que señala su gran extensión espacial. En términos generales, la temperatura decrece desde el centro a la periferia de la ciudad, más allá de la circunvalación de Américo Vespucio. Por otro lado, es posible identificar, el efecto sobre la disminución de las temperaturas del aire ejercido por los usos agrícolas de los suelos que conforman el cono de aproximación del ex Aeropuerto de Cerrillos y por algunos parches similares ubicados hacia el sur de la ciudad. Sin embargo, aún en la periferia urbana, se observan islotes de calor causados por la presencia de superficies altamente impermeabilizadas, como los estacionamientos, edificios y pistas de aterrizaje del Aeropuerto Internacional de Santiago.

En términos comparativos, el sector oriente de la ciudad se observa más frío, con temperaturas similares a los sectores no urbanos, es decir entre $18^{\circ} \mathrm{C}$ y $20,5^{\circ} \mathrm{C}$. Ello es observado desde Av. Vicuña Mackenna hacia el este y resulta aún más significativo hacia el oriente de la Avenida Tobalaba, es decir sobre el piedemonte andino. Esta área corresponde al sector de mayor altitud de la ciudad, que a esta hora no recibe radiación solar directa - debido al efecto de sombra de la cordillera de los Andes- y posee una menor densidad de construcciones y mayores porcentajes de vegetación.

Por otro lado, se distinguen áreas de menor temperatura en las zonas de mayor extensión cubiertas con vegetación, que se localizan sobre áreas verdes del centro y poniente de la ciudad, tales como el Parque O’Higgins y la Quinta Normal. No se observan a esta escala diferencias entre las temperaturas aledañas a los cursos de agua y las de la matriz urbana propiamente tal. Por el contrario, el curso del río Mapocho que atraviesa la ciudad de oriente a poniente, se presenta con temperaturas más elevadas, sobre todo al cruzar por el centro y poniente de la ciudad, probablemente debido a las condiciones 
fuertemente artificializadas del lecho, el bajo caudal que exhibe en enero y por contener en su vecindad una autopista urbana. La intensidad máxima de la isla de calor a esta hora del día es de $7^{\circ} \mathrm{C}$.

Figura $\mathrm{N}^{\circ} 2$

Distribución espacial de la temperatura del aire a las 9 hrs. de un día de verano

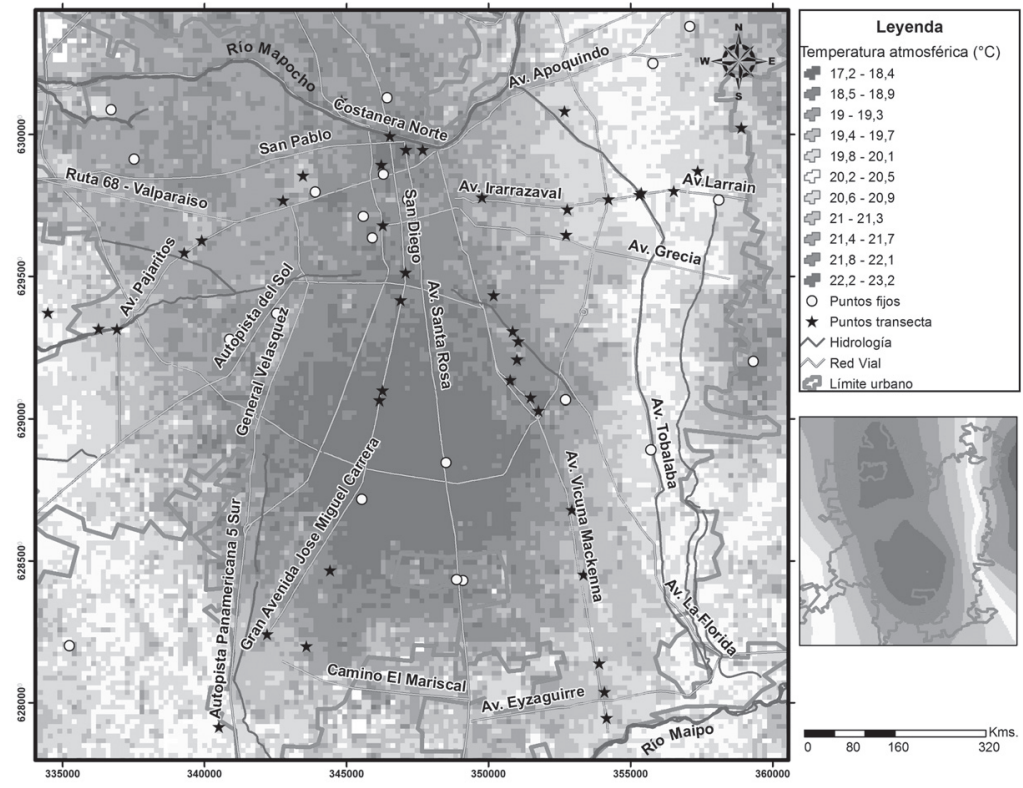

Fuente: Elaboración propia. Mapa ubicado al lado derecho de la figura central corresponde al resultado de la interpolación de la temperatura atmosférica utilizando el método Kriging.

Distribución espacial de la temperatura del aire a las 16:00 horas de un día de verano

Durante la tarde aumentan las diferencias en la distribución espacial de las temperaturas dentro de la ciudad (Figura $\mathrm{N}^{\circ} 3$ ). Gran parte de esta registra valores sobre $30^{\circ} \mathrm{C}$, trasladándose la isla de calor desde el poniente hacia el norte, con temperaturas superiores a $34^{\circ} \mathrm{C}$. La diferencia entre las temperaturas urbanas y rurales es ahora más elevada, por lo que la intensidad de la isla de calor alcanza alrededor de $10^{\circ} \mathrm{C}$.

Al interior de la ciudad existen lugares comparativamente más fríos, con temperaturas similares a aquellas que presentan los sectores rurales, localizados en el sur y oriente. Sigue siendo importante la Avenida Vicuña Mackenna como límite entre zonas termales urbanas, lo que se acentúa en el caso de la Avenida Tobalaba.

Aun al mediodía es posible distinguir el efecto de enfriamiento del cono de aproximación del aeropuerto de Cerrillos, que a esta hora además permitiría el ingreso de masas de aire que ventilen la ciudad debido a la dirección suroeste predominante de los vientos. Se sigue presentando el gradiente de disminución de la temperatura desde el centro a la periferia, con la excepción del nor-poniente de la ciudad. Es en esta última zona donde se localiza la isla de calor urbano.

Si bien ya en la mañana se observa una pequeña isla de calor, restringida al área cercana al Aeropuerto Internacional, es a esta hora que alcanza una extensión mucho mayor, incluyendo áreas no urbanas, con temperaturas superiores a $33^{\circ} \mathrm{C}$. 
Se distingue con gran nitidez el efecto de isla fría desempeñado por los parques al interior de la ciudad. La Quinta Normal alcanza hasta $-3^{\circ} \mathrm{C}$ de diferencia con la matriz urbana circundante y el Parque $\mathrm{O}^{\prime}$ Higgins es $2^{\circ} \mathrm{C}$ más frío. El cerro San Cristóbal es también una isla de frescor alcanzando hasta $-4^{\circ} \mathrm{C}$ de diferencia respecto al área construida ubicada al norponiente de la ciudad.

Figura $\mathrm{N}^{\circ} 3$

Distribución espacial de la temperatura del aire a las 16 hrs. de un día de verano
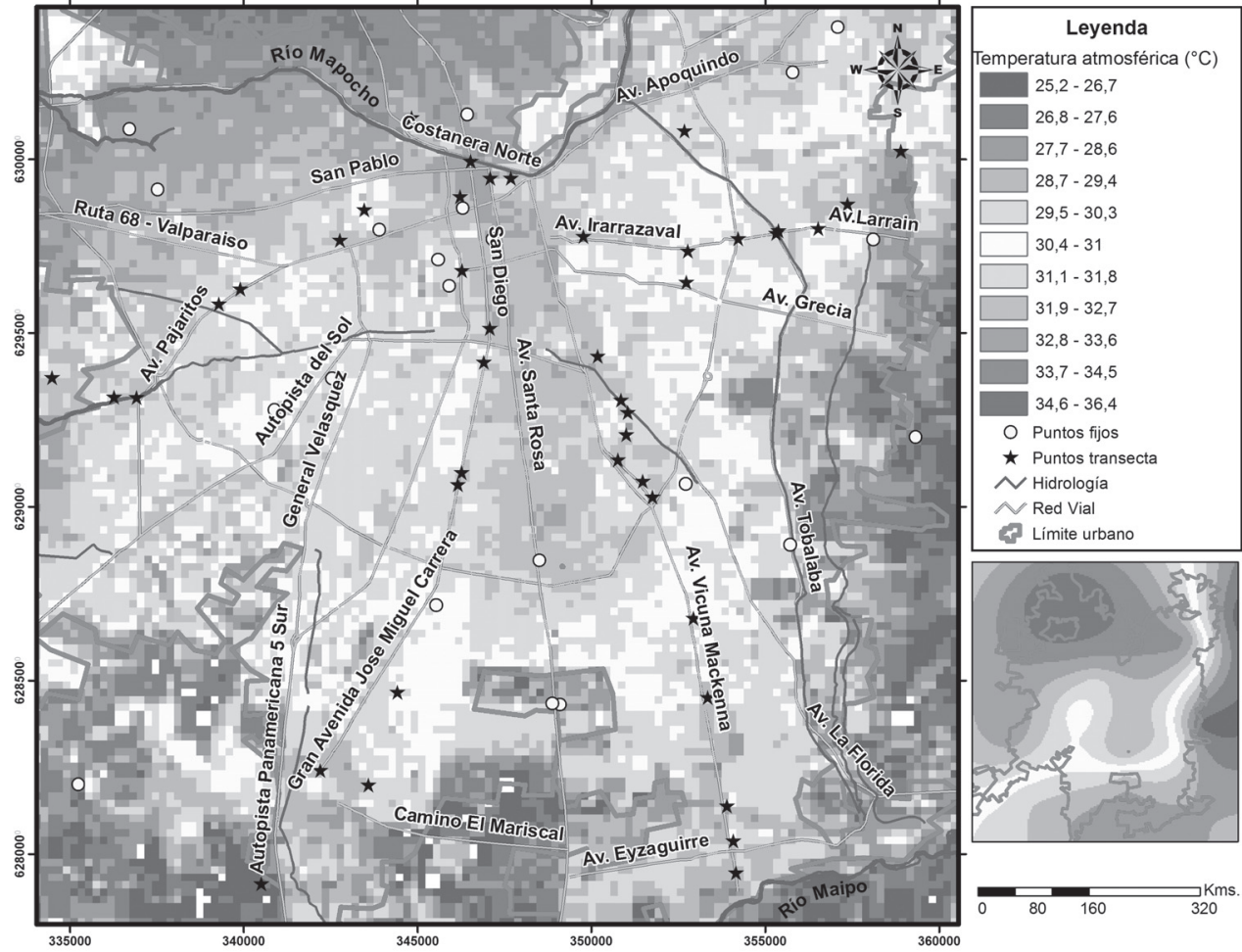

Fuente: Elaboración propia. Mapa ubicado a la derecha de la figura central corresponde al resultado de la interpolación de la temperatura atmosférica utilizando el método Kriging.

\section{Distribución de las temperaturas a las 22:00 horas}

Como muestra la Figura $\mathrm{N}^{0} 4$, unas horas después de la puesta de sol, la ciudad de Santiago se ha convertido en una gran $y$ única isla de calor urbano que incluye casi por completo su superficie urbana, existiendo zonas cálidas también en las áreas rurales ubicadas más allá de su borde norte. En el centro de la ciudad se encuentra una isla que posee la mayor temperatura y que se propaga sutilmente hacia el oriente, aledaña al cerro San Cristóbal.
La diferencia que existe entre los sectores más cálidos y los más fríos de la ciudad y su entorno no urbano alcanza a $10^{\circ} \mathrm{C}$. Incluso durante la noche el sector oriente presenta temperaturas elevadas. La distribución térmica es a esta hora altamente homogénea, ya que no se observa la distinción oriente - poniente, ni las diferencias entre las áreas vegetadas y las construidas. Las construcciones y calles de la ciudad han acumulado gran cantidad de calor durante el día, con temperaturas superiores a $30^{\circ} \mathrm{C}$ por varias horas, y han comenzado, luego de la puesta de sol, a devolverlo a la atmósfera. La vegetación no logra tener 
un efecto significativo sobre la mitigación de las altas temperaturas que resultan evidentes a esta escala.

La diferencia de temperatura con las áreas rurales del sur de la ciudad es igualmente importante a esta hora del día, ubicándose las menores temperaturas sobre el cono de aproximación del aeropuerto de Cerrillos y en partes de las comunas de La Pintana y Puente Alto. Esta diferencia respecto a los sectores rurales del poniente y norte de la ciudad puede deberse al predominio de los vientos suroeste que circulan en la misma dirección que posee el cono de aproximación, y a la presencia del río Maipo, que debiera favorecer un flujo de circulación mayor de vientos.

Figura $\mathrm{N}^{\circ} 4$

Distribución espacial de la temperatura del aire a las 22 hrs. de un día de verano

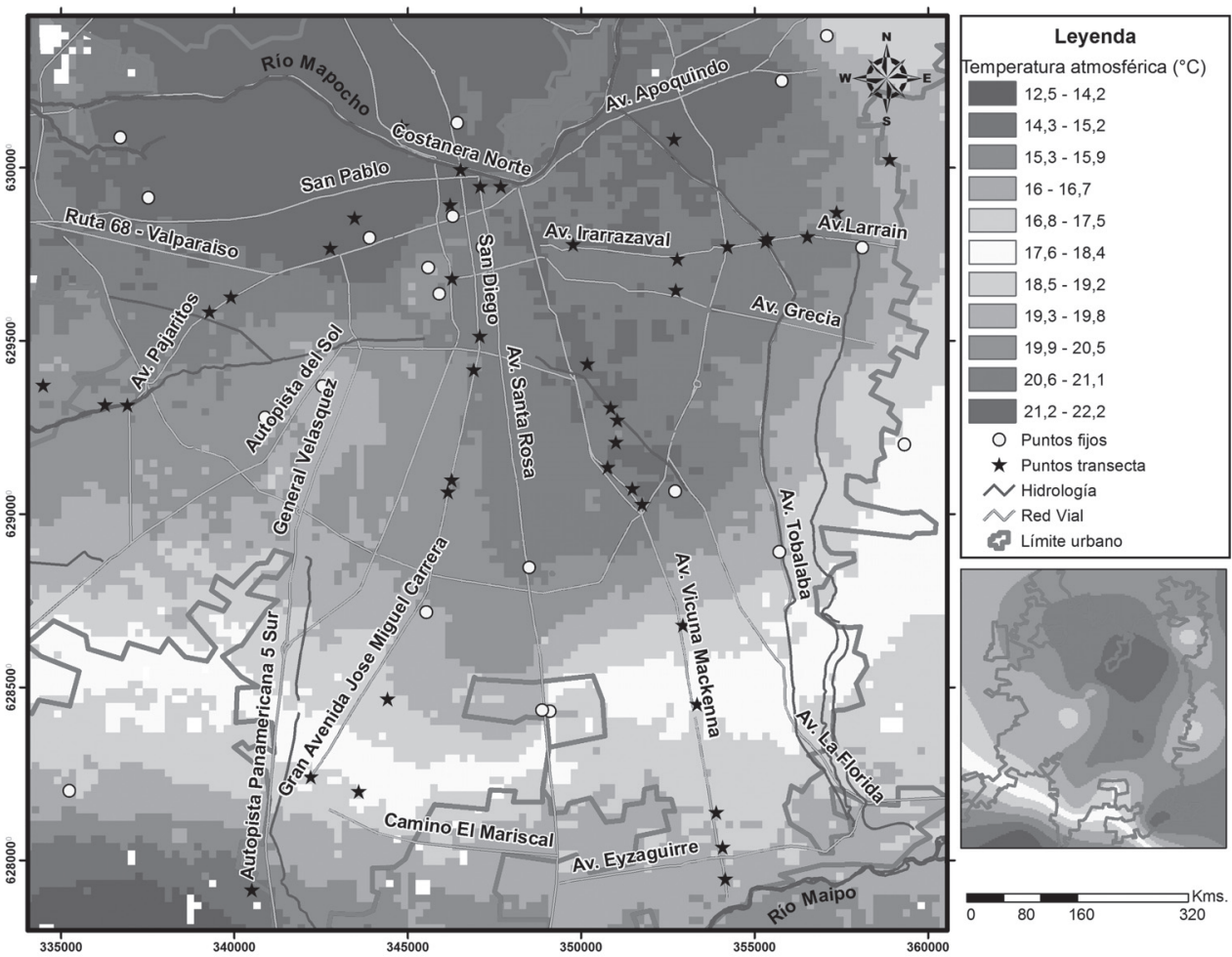

Fuente: Elaboración propia. Mapa ubicado a la derecha de la figura central corresponde al resultado de la interpolación de la temperatura atmosférica utilizando el método Kriging.

\section{Diagnóstico del modelo de regresión}

En la medición de la mañana la diferencia promedio entre los datos observados, los medidos durante los transectos móviles del día 21 de enero de 2009 y los obtenidos con el modelo de regresión múltiple construido alcanzó a $-0,42^{\circ} \mathrm{C}$. De los 49 puntos de mediciones comparados, 34 presentan valores inferiores a un grado de diferencia de temperatura. De los puntos restantes, cinco poseen diferencias superiores a $2^{\circ} \mathrm{C}$ y se localizan, según se observa en la figura 5 , en el poniente y centro de la ciudad, cercanos a varias estaciones meteorológicas fijas. La diferencia puede deberse en este caso a una variabilidad espacial mayor de los datos que no alcanza a ser registrada por los instrumentos de medición fijos pero que sí ha sido medida en terreno. 
Durante la tarde, las diferencias entre los valores estimados y observados son menores. Cuarenta y dos de los cuarenta y nueve puntos evaluados poseen diferencias inferiores a un grado Celsius. Solo tres puntos del modelo de regresión poseen diferencias superiores a los $2^{\circ} \mathrm{C}$, estando ubicados el primero en el centro de la comuna de Quilicura (al norte de la ciudad), lugar que había presentado también diferencias de este orden durante la mañana; los dos puntos restantes se localizan más al sur, uno de ellos en la ribera norte del río Maipo, área que no se encuentra representada por estaciones meteorológicas fijas.

En la noche se observan las mayores diferencias cuando se comparan los datos modelados y los datos observados, que superan los $3,5^{\circ} \mathrm{C}$ e incluso alcanzan a $10^{\circ} \mathrm{C}$. La diferencia promedio es de $1,7^{\circ} \mathrm{C}$.

De los 49 puntos registrados, 33 poseen diferencias por debajo de un grado; de los restantes, 16 presentan diferencias superiores a $2{ }^{\circ} \mathrm{C}$, localizados en el centro y sur de la ciudad, cercanos a estaciones meteorológicas fijas. De los cinco puntos que poseen más de $3{ }^{\circ} \mathrm{C}$ de diferencia, dos se ubican en la periferia, uno por el oriente y otro en el sur de la ciudad. Cuatro son los puntos con $5^{\circ} \mathrm{C}$ de diferencia, tres de los cuales se ubican al norte del río Maipo, que como se ha dicho antes, corresponde a un lugar sin estaciones fijas. El punto restante se localiza en San Bernardo. Con más de $10^{\circ} \mathrm{C}$ existe un único punto ubicado en la ribera sur del Maipo, en un sector predominantemente rural.

Figura $\mathrm{N}^{\circ} 5$

Diferencias encontradas entre los datos de temperatura modelados y los datos observados, en cada punto de las transectos móviles realizadas el 21 de enero de 2009

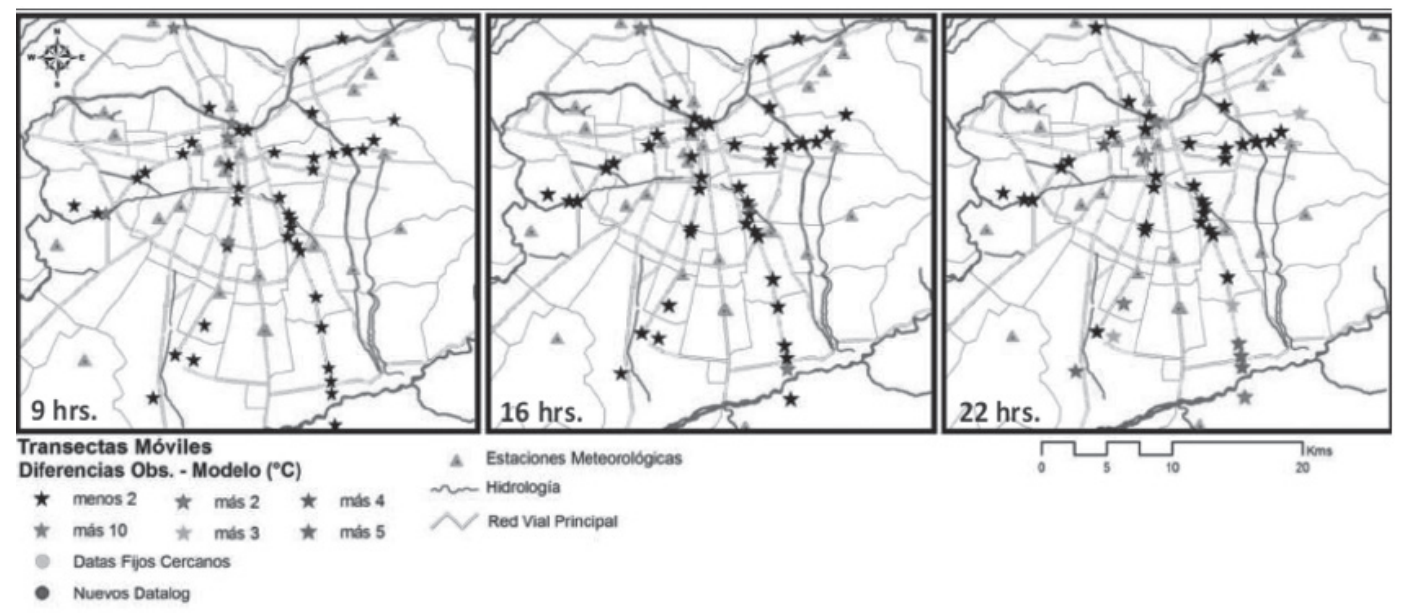

Fuente: Elaboración propia.

\section{Factores de la distribución espacial de la temperatura}

La ciudad de Santiago genera importantes islas de calor urbano y sus niveles de impermeabilización de los suelos contribuyen a elevar las temperaturas del aire en forma significativa. Por el contrario, las coberturas de vegetación, constituyen uno de los más im- portantes elementos para mitigar este proceso de calentamiento y controlar la extensión y magnitud de las islas de calor urbano (Honjo et al., 2003; Nowak \& McPherson, 1993). En la ciudad de Santiago es posible afirmar que las superficies cubiertas de vegetación son capaces de disminuir hasta en $1,5^{\circ} \mathrm{C}$ la temperatura atmosférica en una tarde de verano, y que se pueden encontrar diferencias 
que superan los $4^{\circ} \mathrm{C}$, entre los parques que se reconocen a escala metropolitana y sus áreas urbanas adyacentes, constituyéndose en auténticas islas de frescor.

A pesar que se ha planteado que la distancia a los cursos de agua, la orientación y la rugosidad de las edificaciones influyen sobre el comportamiento de la temperatura atmosférica, los resultados obtenidos en este trabajo y a esta escala espacial, no han permitido su reconocimiento como factores significativos. Es probable que la escasa incidencia de la rugosidad se explique por la no consideración de brisas y vientos locales, que según Cuadrat et al. (2003) y Alcoforado et al. (2009) actuarían como factores relevantes de los climas urbanos. Además, la escala y calidad de la información obtenida respecto de la altura de las edificaciones en la ciudad es muy limitada.

Se puede advertir que el crecimiento de las superficies urbanizadas y el reemplazo de coberturas naturales y seminaturales por usos urbanos (Sudhira et al., 2004; Xian \& Crane, 2005, entre otros), contribuyen a originar particularidades climáticas caracterizadas no solo por ser más cálidas, sino que también por su alta heterogeneidad espacial, constituyendo un mosaico de paisajes fuertemente influenciados por el diseño y su materialidad, en los cuales sobresale la existencia de islas de calor urbano. Estas últimas, en la estación de verano, no corresponden necesariamente al modelo espacial de gradiente térmico presentado por Oke (1987), en la medida que las mayores temperaturas no siempre se localizan en las áreas centrales de la ciudad. Ello se debería a las diferencias que existen entre las ciudades latinoamericanas, esencialmente difusas y policéntricas, respecto de la configuración espacial de las ciudades europeas, esencialmente unicentrales y compactas, que han servido como fuente para la realización de modelos de gradientes térmicos. A mayor abundamiento, durante las tardes de verano, se registran también en Santiago, amplias superficies ocupadas por islas de calor en sectores no urbanos localizados al norponiente, respecto a los cuales sería posible considerar a la ciudad como una zona de hundimiento térmico (Peña y Romero, 2006), una condición específica de ciudades desarrolladas sobre paisajes áridos y semiáridos (Comrie, 2000; Correa et al., 2006 y 2010).

Las islas de calor ubicadas al poniente de la ciudad, ocupando superficies no urbanas, corresponden a terrenos industriales y áreas naturales desforestadas, a lo que se debe agregar el color obscuro de los sedimentos, producto de su formación geológica cinerítica. El sector norponiente además se ubica sobre los sectores más bajos de la cuenca, favoreciendo la concentración de brisas de aire cálido que actuan aumentando las temperaturas (Cuadrat et al., 2005; Jo et al., 2001; Romero et al., 2008).

Por el contrario, las temperaturas más bajas se registran en la zona SW de la ciudad. Las comunas de San Bernardo y Calera de Tango, concentran masas de aire menos cálidas que podrían ser transferidas al centro de la ciudad a través del corredor formado por el cono de aproximación del aeropuerto de Cerrillos, considerando una similar dirección predominante de los vientos.

Esta investigación considera unicamente información proporcionada por estaciones meteorológicas de propiedad del sector público y con acceso gratuito, lo que se puede considerar un bien público propiamente tal. Dicha información, sin embargo, no está contenida en una red coordinada, sino que por el contrario, corresponde a estaciones instaladas por diversas instituciones y por consiguiente, con objetivos también diversos, lo que impone una limitación al estudio. Existen amplias zonas de la ciudad no cubiertas por estaciones meteorológicas fijas y dudas respecto a la calidad de los datos, que pueden ser parte importante de la causa de las diferencias observadas entre los resultados modelados y medidos en terreno para la noche del 21 de enero de 2009.

Respecto a lo mismo, y debido a las particularidades observadas en el comportamiento climático de la ciudad de Santiago, no es posible recomendar la replicación de sus resultados en otras ciudades, sobre todo si se consideran los factores que influyen mayormente en la distribución de las temperaturas del aire. 
La utilización de información proporcionada por imágenes de satélite permitió la obtención de representaciones espaciales continuas, siendo un complemento significativo para la interpretación de la información discreta proveniente de estaciones meteorológicas fijas y móviles, tal como lo plantean Voogt \& Oke (2003), Beeson et al. (2005) y Farías (2007), entre otros.

\section{Conclusiones}

De los ocho factores de distribución espacial de las temperaturas del aire al interior de la ciudad, mencionados por la literatura, en el caso de Santiago durante las horas de la mañana y tarde de un día de verano, los modelos de regresión permiten reconocer por sus funciones explicativas a las coberturas vegetales, superficies impermeables, altura, las temperaturas de emisión superficial del suelo y la estructura espacial del campo térmico representada por la interpolación de los datos. Durante las noches, los factores explicativos mayores se reducen a los dos primeros y a la estructura espacial del campo térmico.

En el transcurso de los días de verano la distribución espacial de las temperaturas del aire varía según la hora y de acuerdo a la incidencia de los factores explicativos. Tal como se había demostrado con anterioridad para las estaciones de otoño e invierno, las áreas más frías de la ciudad se distribuyen en su sección oriental. Las más cálidas lo hacen en el sector sur.

El sector oriente de Santiago puede registrar temperaturas similares a las de las áreas rurales, relevando el efecto de enfriamiento que deben jugar las brisas de montaña a valle generadas en el piedemonte andino, que en consecuencia se constituye en una de las zonas de protección de la calidad climática de la ciudad. Sin embargo, dicho rol o el de otras superficies de enfriamiento debería acentuarse para que la isla de calor urbano no se generalizara sobre la ciudad, como sucede actualmente en las noches de verano en la totalidad de Santiago, cuando la metrópolis se transforma en una superficie continua de calor.
El que existan factores ambientales y urbanos que están influenciando los comportamientos térmicos de la ciudad constituyen antecedentes valiosos de considerar en su planificación y gestión ambiental y proporcionan argumentos que permitirían definir acuerdos, y formular e implementar políticas públicas urbanas que aseguren la sustentabilidad ambiental de la ciudad de Santiago, en particular para enfrentar el efecto del calentamiento global que predicen los modelos de cambio climático. Más allá de ello, una ciudad ambientalmente sustentable debe demostrar su capacidad de mitigar las concentraciones de calor y sus implicancias sobre el disconfort térmico y pérdida de calidad de vida que pueden sufrir sus habitantes, por un lado, como las relaciones con la calidad del aire, la contaminación atmosférica y sus efectos adversos sobre la salud de sus habitantes. Las ciudades modernas intentan desarrollar sus planes de urbanización siguiendo conceptos que sean compatibles con la naturaleza y empleando diseños, técnicas y materiales que optimicen el uso de las fuentes naturales de calor, humedad y ventilación, reduciendo de esta manera la dependencia de energías importadas al medio urbano.

Es importante aumentar el conocimiento de la climatología urbana e incorporar sus aportes en la planificación y gestión ambientalmente sustentable de las ciudades chilenas en general y de la ciudad de Santiago en particular, especialmente en la medida que su población, cercana a los siete millones de habitantes, involucra a un $45 \%$ del total nacional, que aspira a niveles de calidad de vida crecientemente mejores. Para superar las actuales limitaciones de conocimiento, se requiere el desarrollo del concepto de red de monitoreo que sirva a la articulación de las diferentes fuentes de datos meteorológicos actualmente disponibles para la ciudad de Santiago. Si bien es cierto, se cuenta con datos provenientes de más de treinta estaciones meteorológicas, el que pertenezca a instituciones distintas que tienen objetivos diversos aumenta las dificultades de coordinación, validación, representatividad temporal y espacial y continuidad de los registros. El monitoreo del clima urbano, como la necesidad de asegurar una adecuada calidad del aire de las ciudades chilenas debe obedecer a una institución comprometida con la mejor condición ambiental de sus paisajes urbanos. 


\section{Referencias bibliográficas}

ALCOFORADO, M.; ANDRADE, H.; LOPES, A. \& VASCONCELOS, J. Application of climatic guidelines to urban planning: The example of Lisbon (Portugal). Landscape and Urban Planning, 2009, No 90, p. 56-65.

ARNFIELD, J. Two Decades of Urban Climate Research: A Review of Turbulence, Exchanges of Energy and Water, and the Urban Heat Island. International Journal of Climatology, 2003, № 23, p. 1-26.

ARNOLD, C. \& GIBBONS, J. Impervious Surface Coverage: The Emergence of a Key Environmental Indicator. Journal of the American Planning Association, 1996, Vol. 62, No 2, p. 243-258.

BEESON, C.; BLUMBERG, D.; BRAZEL, A. \& SHASUA-BAR, L. Combined methods of thermal remote sensing and mobile climate transects in Beer Sheva, Israel. International Archives of Photogrammetry, Remote sensing and Spatial Information Sciences, 2005, Vol. XXXVI, Part8/W27.

BOLUND, P. \& HUNHAMMAR, S. Ecosystem services in urban areas. Ecological ECOnomics, 1999, No 29, p. 293-301.

COMRIE, A. Mapping a Wind-Modified Urban Heat Island in Tucson, Arizona. Bulletin of the American Meteorological Society. 2000, Vol. 81, No 10, p. 2417- 2426.

CORREA, E.; RUIZ, M. y CANTÓN, M. Morfología forestal y confort térmico en "ciudades oasis" de zonas áridas. Ambiente construido, 2010, Vol. 10, No 4, p. $119-127$.

CORREA, E.; DE ROSA, C. \& LESINO, G. Isla de calor urbana. Distribución espaciotemporal de temperaturas dentro del área Metropolitana de Mendoza. Revista Avances en Energías Renovables y Medio Ambiente, 2006, Vol. 10, No 11, p. 121-128.

COUTTS, A.; BERINGER, J.; JIMMI, S. \& TAPPER, N. The urban heat island in Melbourne: drivers, spatial and temporal variability, and the vital role of stormwater. 2007. Disponible en internet: https://www. clearwater.asn.au/user-data/resource-files/ urban-heat-island-in-melobourne2009.pdf

CUADRAT, J.; SAZ, M. \& VICENTE-SERRANO, S. Surface wind direction influence on spatial patterns of urban heat island in Zaragoza (Spain). Geophysical Research Abstracts, 2003, No 5, 02592.

CUADRAT, J.; VICENTE- SERRANO, S. y SAZ, M. Los efectos de la urbanización en el clima de Zaragoza (España): La isla de calor y sus factores condicionantes. Boletín de la A.G.E, 2005, $\mathrm{N}^{\circ} 40$, p. 311-327.

DEPARTAMENTO DE GEOFÍSICA UNIVERSIDAD DE CHILE (DGF). Estudio de la variabilidad climática en Chile para el siglo $X X I$. Santiago de Chile: Comisión Nacional del Medioambiente (CONAMA), 2006.

ELIASSON, I. The use of climate knowledge in urban planning. Elsevier, Landscape and urban planning, 1999, № 48, p. 31-44.

FARÍAS, G.; DESSI, A.; MASELLI, F. y CROTTI, C. Integración de series de datos satelitales y climáticos para la definición de áreas climáticamente homogéneas en el centro -nordeste de Argentina, 2007. Disponible en internet: hum.unne.edu.ar/revistas/ geoweb/geo4/archivos/farias.pdf

FLOCKS, J.; ESCOBEDO, F.; WADE, J.; VARELA, S. \& WALD, C. Environmental Justice Implications of Urban Tree Cover in Miamii Beach County, Florida. Environmental Justice, 2011, Vol. 4, No 2, p. 125-134.

GEORGAKIS, C.; SANTAMOURIS, M. \& KAISARLIS, G. The vertical stratification of air temperature in the center of Athens. Journal of Applied Meteorology and Climatology, 2010, No 49, p. 1219-1232.

GRIMMOND, S. \& OKE, T. Heat storage in urbana areas: local scale observation and evaluation of a simple model. Journal of Applied Meteorology, 1998, No 38, p. 922940.

HULME, M. Climate and its changes: a cultural appraisal. Geography and Environment, 2015, № 2, p. 1-11. 
JO, M.; LEE, K.; JUN, B.; KWON, B. \& JO, Y. The spatial topographic of urban surface temperature using remotely sensed data and GIS. In: $22^{\text {nd }}$ Asian conference on remote sensing, Singapore, 2001.

JOHANSSON, E. Influence of urban geometry on outdoor thermal comfort in a hot dry climate: a study in Fez, Morocco. Building and Environment, 2006, No 4, p. 1326-1338.

HONJO, T.; NARITA, K-I.; SUGAWARA, H.; MIKAMI, T.; KIMURA, K. \& KUWATA, N. Observation of cool island effect in urban park (Shinjuku Gyoen). In: 15 ${ }^{\text {a }}$ Conferencia Internacional de Clima Urbano, 1- 5 Sept., Polonia, 2003.

IRARRÁZABAL, F. Clasificación y evaluación ambiental de zonas termales en el transecto entre las comunas de Pudahuel y Las Condes y su relación con el mercado inmobiliario. Santiago de Chile: Memoria para optar al Título Profesional de Geógrafo, Facultad de Arquitectura y Urbanismo, Universidad de Chile, 2011.

KUSAKA, H. Recent progress in urban climate study in Japan. Geographical Review of Japan, 2008, Vol. 81, No 5, p. 361-374.

LINÉS, A. Contribución al concepto de clima. Territoris, 1996, № 1, p. 201-213.

MOLINA, M. Efectos de los tipos de urbanización asociados al crecimiento urbano del área Metropolitana del Gran Santiago sobre la generación y comportamiento de micro islas de calor. Santiago de Chile: Memoria para optar al Título Profesional de Geógrafo, Facultad de Arquitectura y Urbanismo, Universidad de Chile, 2007.

MOLINERO, L. Construcción de modelos de regresión multivariantes. Asociación de la Sociedad Española de la Hipertensión, 2002. Disponible en Internet: www.ccp.ucr. ac.cr/ icamacho/bioest103/contenido/regresion1.pdf

NOWAK, D. y MCPHERSON, E.G. Cuantificación del impacto ambiental de los árboles en Chicago. Unasylva, 1993, Vol. 44, No 173, p. XX-XX
NYUK, W. \& KARDINAL, S. GIS-based greenery evaluation on campus master plan. Journal Landscape and Urban Planning, 2008, No 84 , p. 166-182.

OKE, T. The energetic basis of the urban heat island. Quarterly Journal of Royal Meteorological Society, 1982, No 108, p. 1-24.

OKE, T. The thermal regime of urban parks in two cities with different summer climates. International Journal of Remote Sensing, 1987, Vol. 19, No 11, p. 2085-2104.

OKE, T. The distinction between canopy and boundary layer urban heat island. Atmosphere, 2010, Vol. 14, № 4, p. 266-277.

PAULEIT, S.; ENNOS, R. \& GOLDING, Y. Modeling the environmental impacts of urban land use and land cover change - a study in Merseyside, UK. Landscape and Urban Planning, 2005, № 71, p. 295-310.

PEÑA, M. y ROMERO, H. Relación espacial y estadística entre las islas de calor de superficie, las coberturas vegetales, la reflectividad y el contenido de humedad del suelo, en la ciudad de Santiago y su entorno rural. Anales de la Sociedad de Ciencias Geográficas de Chile, 2006, p. 107-117.

PRASHAD, L. Urban Materials and Temperature in Phoenix: Connecting Land Use, Socioeconomics, and Vegetation and Relating Ground and Air Variables. Tucson: M.S. Thesis Defense, Arizona State University, 2004.

ROHINTON, E. Urban Heat Island \& Cooling Load: The case of an Equatorial City. Architecture, Energy \& Environment, Vol. 16, $\mathrm{N}^{\mathrm{o}} 1$, p. 16-18.

ROMERO, H.; MOLINA, M.; MOSCOSO, C.; SARRICOLEA, P. y VÁSQUEZ, A. Cambio climático y sustentabilidad urbana de las metrópolis chilenas. En: /I Coloquio Chile Metropolitano, Universidad del Bío-Bío, Concepción, 20-21 noviembre, 2008.

ROMERO, H. y OPAZO, D. Ecología política de los espacios urbanos metropolitanos: Geografía de la Justicia ambiental. Revista Geográfica de América Central, Número especial EGAL 2011, p. 1-16. 
SALAMANCA, F. Desarrollo de modelos numéricos para investigar la isla de calor en ciudades y estudio de la sensibilidad de distintos parámetros urbanos. Madrid: Tesis para optar al grado de Doctor, 2010.

SARRICOLEA, P. Análisis de la sustentabilidad del crecimiento urbano de la ciudad de Santiago y sus efectos sobre la configuración de las temperaturas superficiales. Santiago de Chile: Tesis para optar al grado de Magíster en Geografía con mención en Recursos Territoriales, Universidad de Chile, 2008.

SARRICOLEA, P. y ROMERO, H. Análisis de los factores condicionantes sobre las temperaturas de emisión superficial en el Área Metropolitana de Valparaíso. A.C.E., 2009, No 14, p. 79-96.

SARRICOLEA, P. La isla de calor urbana de superficie y sus factores condicionantes: el caso del Área Metropolitana de Santiago. Barcelona: Memoria para optar al grado de Doctor, Universidad de Barcelona, 2012.

SENDIÑA, I. y PÉREZ, V. Fundamentos de Meteorología. Santiago de Compostela: Servicio de Publicaciones e Intercambio Científico, Universidad de Santiago de Compostela, 2006.

SPROKEN - SMITH, R. \& OKE, T. Scale modeling of nocturnal cooling in urban parks. Boundary Layer Meteorology, 1999, № 93, p. 287-312.
STEWART, I.D. \& OKE, T. Classifying urban climate field sites by local climate zones: The case of Nagano, Japan. In: Preprint, Seventh International Conference on Urban Climate, 29 june -3 july 2009, Yokohama.

SUDHIRA, H.S.; RAMACHADRA, T.V. \& JAGADISH, K.S. Urban sprawl: metrics, using GIS. International Journal of Applied Earth Observation and Geoinformation, 2004, № 5, p. 29-39.

VILLARROEL, C.; ROSENBLUTH, B. \& ACEITUNO, P. Climate change along the extratropical west coast of south America (Chile): daily $\mathrm{max} / \mathrm{min}$ temperatures. In: Proceedings of $8^{\circ}$ Conference on Southern Hemisphere Meteorology and Oceanography (ICSHMO), Foz de Iguazu, Brasil, 2006, p. 487-489.

VOOGT, J.A. \& OKE, T.R. Thermal remote sensing of urban climates. Remote Sensing of Environment, 2003, № 86, p. 370-384.

WONG, M.; NICHOL, J. \& KAWOK, K. The urban heat island in Hong Kong: causative factors and scenario analysis. Urban remote sensing joint event, 2009. Disponible en Internet: http://ieeexplore.ieee.org/stamp/stamp. jsp?arnumber $=05137468$.

XIAN, G. \& CRANE, M. Assessments of urban growth in the Tampa Bay watershed using remote sensing data. Remote Sensing of Environment, 2005, № 97, p. 203-215. 
